\title{
Efficient Inter-Process Synchronization for Parallel Discrete Event Simulation on Multicores
}

\author{
Pavol Bauer, Jonatan Lindén, Stefan Engblom and Bengt Jonsson \\ Dept. of Information Technology, Uppsala University \\ \{pavol.bauer,jonatan.linden,stefane,bengt\}@it.uu.se
}

\begin{abstract}
We present a new technique for controlling optimism in Parallel Discrete Event Simulation on multicores. It is designed to be suitable for simulating models, in which the time intervals between successive events between different processes are highly variable, and have no lower bounds. In our technique, called Dynamic Local Time Window Estimates (DLTWE), each processor communicates time estimates of its next inter-processor event to (some of) its neighbors, which use the estimates as bounds for advancement of their local simulation time. We have implemented our technique in a parallel simulator for simulation of spatially extended Markovian processes of interacting entities, which can model chemical reactions, processes from biology, epidemics, and many other applications. Intervals between successive events are exponentially distributed, thus having a significant variance and no lower bound. We show that the DLTWE technique can be tuned to drastically reduce the frequency of rollbacks and enable speedups which is superior to that obtained by other works. We also show that the DLTWE technique significantly improves performance over other existing techniques for optimism control that attempt to predict arrival of inter-process events by statistical techniques.
\end{abstract}

\section{Categories and Subject Descriptors}

I.6.8 [Simulation and Modeling]: Types of simulationDiscrete Event, Parallel

\section{Keywords}

Parallel Discrete-Event Simulation, PDES, Optimism control, Multicore, Spatial Stochastic Simulation

\section{INTRODUCTION}

Discrete Event Simulation (DES) is an increasingly important tool for evaluating system models in all fields of

Permission to make digital or hard copies of all or part of this work for personal or classroom use is granted without fee provided that copies are not made or distributed for profit or commercial advantage and that copies bear this notice and the full citation on the first page. Copyrights for components of this work owned by others than ACM must be honored. Abstracting with credit is permitted. To copy otherwise, or republish, to post on servers or to redistribute to lists, requires prior specific permission and/or a fee. Request permissions from permissions@ acm.org. SIGSIM-PADS'15, June 10-12, 2015, London, United Kingdom. Copyright (C) 2015 ACM 978-1-4503-3557-7/15/06 ...\$15.00. DOI: http://dx.doi.org/10.1145/2769458.2769476. science and engineering. To improve the capacity and performance of DES simulators, several techniques for Parallel DES (PDES) were developed in the 90's [26, 20, 25, 15]. Parallelization made it possible to simulate large system models, but it was challenging to achieve good speedup corresponding to the number of employed processors. A major difficulty was that PDES requires fine-grained synchronization between processing elements, which was not easy to realize efficiently on multiprocessors at that time, given the comparatively long communication delays between processing elements. With the current advent of multicore processors, these delays have decreased, triggering the development of new techniques for PDES targeting multicores (e.g., [7, 27, $35])$.

In PDES, the simulation model is partitioned onto logical processes (LPs), each of which evolves its sub-model along a local simulation time axis. LPs exchange timestamped events to incorporate inter-LP dependencies. Each LP must ensure that the processing of incoming events is correctly interleaved with local events. The problem with incoming events that violate an LP's local timestamp ordering (socalled stragglers) can in principle be handled in two ways: conservative approaches allow an LP to process an event only when it is guaranteed that no straggler will later arrive [26]; optimistic approaches allow stragglers by invoking suitable corrective action (rollback) $[20,25]$. In purely conservative approaches, local execution of LPs may be blocked excessively unless inter-LP events can always be predicted long in advance (e.g., when simulating networks with long communication latencies), which most often is not possible. On the other hand, in optimistic approaches, performance may be damaged by excessive numbers of rollbacks. Many approaches to PDES therefore allow stragglers, but control the optimism by various heuristic techniques, based on, e.g., observed frequency of rollbacks [29, 8], patterns of past interLP messages [14], etc.

In this paper we present a new technique for controlling optimism in PDES. It is particularly designed for high efficiency when simulating models in which the time intervals between successive inter-LP events are highly variable and have no lower bounds. Such models pose severe difficulties for both conservative and existing variants of optimistic approaches. Our technique, called Dynamic Local Time Window Estimates (DLTWE), exploits the opportunities for fast multicore inter-LP communication. DLTWE assumes that an LP can reasonably estimate timestamps of its next $k$ outgoing inter-LP events, where $k$ is a tuneable parameter of our technique. Each LP continuously communicates these 
estimates to its corresponding neighboring LPs, which use the estimates as bounds for advancing their local simulation time. Since the communicated timestamps are merely estimates, DLTWE does not rule out the occurrence of stragglers, meaning that each LP must perform rollbacks when needed. If the estimates are sufficiently accurate, then the number of rollbacks should be small, allowing the simulator to operate with high efficiency.

We have developed the DLTWE technique in the context of stochastic spatial simulation of models governed by the mesoscopic reaction-diffusion master equation (RDME) [4]. Here the model's geometry is discretized into small subvolumes (a.k.a., voxels), each of which contains a discrete number of species (e.g., molecules). In each subvolume the species obey prescribed stochastic reaction laws and the species may move (by diffusion) to other neighboring subvolumes. When simulating an RDME model using PDES, the subvolumes are partitioned onto LPs. Hence, a diffusion event between two boundary subvolumes causes interLP communication. By the Markovian nature of the model, the waiting time of any event is an exponentially distributed random variable; thus the waiting time has a significant variance and no lower bound.

In the present paper we show that the DLTWE technique has small overhead when implemented on a shared-memory multicore processor. In our simulator, each LP maintains a list of future events, whose occurrence times have already been sampled; this is already a component of our technique for simulating RDME models [3]. We show how the DLTWE technique can be tuned by limiting how far into the future DLTWE estimates will be provided: The cost of providing more accurate DLTWE estimates further into the future can be tuned, both against the cost of rollbacks caused by poor estimates and against achieving limited optimism in contexts where rollbacks are relatively inexpensive.

In the paper, we also demonstrate the effect of a technique to limit the cost of rollbacks by reversing only those processed events that are causally dependent on the straggler that caused the rollback in the first place. Less costly rollbacks also allow more optimism in the simulation, thereby limiting waiting and increasing overall simulation efficiency.

In our evaluation, we show how our implementation of the DLTWE technique enables speedups in parallel simulation of RDME models, which is superior to that obtained by other works. We support this comparison by a detailed profiling of the simulator behavior, which shows how DLTWE significantly reduces both the cost of unnecessary blocking and of excessive rollbacks. In particular, we compare the DLTWE technique against other existing techniques for optimistic control, such as the Probabilistic Adaptive Direct Optimism by Ferscha [14], and show that employing the DLTWE results in at least a doubling of parallel efficiency.

After reviewing related work in the next section, we review the class of spatial stochastic simulation models considered by our simulator in Section 3. A detailed description of our parallelization algorithm, including the DLTWE technique, is given in Section 4. Section 5 contains a detailed evaluation of the performance of our parallelization technique, including a detailed breakdown of the simulation effort, and a comparison with other techniques for optimism control. Section 6 contains conclusions and directions for future work.

\section{RELATED WORK}

Numerous methods for synchronization in PDES have been proposed. Extensive surveys are provided in [15, 9, 18]; here we can only review a selection.

Synchronization methods can be coarsely classified into conservative [26] and optimistic [20, 25]. Each approach has its drawbacks, which subsequently proposed techniques aim to mitigate. For instance, conservative time windows [1] are used to increase parallelism in a purely conservative approach: this assumes that there is always a guaranteed lower bound on the delay until the next inter-LP event, which does not exist in stochastic simulations that we consider.

Optimistic approaches [20,25] have the potential to achieve higher parallelism, but performance may be damaged by excessive rollbacks. Many techniques have been developed for controlling the optimism and limiting the frequency of rollbacks. One idea is to employ dynamically moving time windows that bound how far each LP can advance its local time (e.g., [31, 33]). Synchronization between time windows typically assumes frequent calculation of global virtual time (GVT), which is an expensive global calculation, for which a special high-speed network is recommended. A further development of these approaches is the class of "nearperfect" state information (NPSI) protocols, including the elastic time algorithm [32]. Here, the bound is based on GVT and information about future messages to neighboring LPs, which is computed and communicated over a special high-speed network. Our DLTWE approach is also based on controlling optimism by information propagated between LPs; however, we show how such an idea can be realized on a modern multicore without using such a high-speed network.

There are also approaches where optimism control can be performed by LPs based on locally available information, not requiring a special high-speed network. In some of these techniques, each LP autonomously regulates its event processing speed against parameters, such as frequency of rollbacks $[29,8]$. Another approach is to use the pattern of past incoming inter-LP messages [14] to predict the time of the next incoming message by statistical techniques, thereby obtaining a bound for advancement of local time. We compare this approach to our technique in Section 5.5. A study where model-specific information has been used to extract additional synchronization information (in the form of an extended lookahead) is presented in [24]. In our approach we also make use of model-specific knowledge; we extract relevant entries from the event queue and communicate them to neighbors as DLTWEs.

Other performance-enhancing techniques in optimistic PDES include to avoid rollbacks due to out-of-timestamporder when this is possible. Chen and Szymanski [6] introduce a "lookback", a limited history of recent events. When a straggler event arrives, it is checked against the history, and if no causality error is found, the event is processed as if arriving in order. Leong et al. [23] view the processes in the simulator as objects of abstract data types, and messages as operations being performed on the objects. Some of the operations commute with each other, and hence rollbacks can be avoided. Recently, techniques for PDES that specifically target multicores have been developed. One approach is to allow each subdomain to be accessed by several cores (e.g., $[7,27])$, thereby achieving better load balancing. 
We conjecture that for our work, this benefit would be more than outweighed by the cost needed for synchronizing accesses to shared data. Wang et al. [35] present a multicore NUMA-aware modification of the general optimistic simulator ROSS [5]. We believe that our simulator would gain from being optimized for a NUMA architecture as well, such an improvement is however orthogonal to what we present here.

Parallel simulation of RDME models using exact numerical methods was previously addressed by $[21,34,10]$. The simulators are implemented in MPI, where each LP is mapped to an MPI process. Each LP simulates a subvolume [10, 34] or a larger subdomain [21]. As discussed by Dematté and Mazza [10], conservative simulation of RDME models is infeasible due to the lack of precise lookahead. Hence, simulators rely on optimistic protocols. A reduction of rollback cost for RDME models was previously implemented by a static time window from the GVT [21] or adaptive protocols, such as Breathing Time Warp [34]. A more general class of diffusive systems can also be represented in CellDEVS, parallel simulation of such models has been studied by Jafer et al. [19].

\section{SPATIAL STOCHASTIC SIMULATION}

In this section, we review the class of spatial stochastic simulation models considered by our simulator.

The reaction-diffusion master equation (RDME) is a framework to describe the dynamics of spatially extended Markovian processes of interacting entities. As the name suggests, the RDME is a suitable model for chemical reactions in a diffusive environment, but processes from biology, epidemics, and many other applications may also be successfully treated. In particular, the RDME is particularly suitable for systems where discrete effects (due to small populations) and thermal noise should not be neglected.

The spatial domain of interest is divided into subvolumes, each of which maintains a copy number (discrete count) of all participating species. The dynamics of the model is then a continuous-time Markov chain over the state space consisting of all copy numbers in all subvolumes. The state transitions fall in one of two categories, (i) a reaction event acts in a single subvolume by removing a combination of species and replacing it with a different combination, (ii) a diffusion event moves a single unit of one species from one subvolume to a neighboring subvolume, and hence changes the state of two subvolumes. The waiting time for each transition is exponentially distributed with an intensity that is proportional to the product of the copy numbers of the involved species.

As a concrete example, a reaction from the Lotka-Volterra predator-prey model described in Section 5.1 reads

$$
B+C \stackrel{r}{\rightarrow} 2 C,
$$

that is, in a particular subvolume one unit of $B$ (prey) is consumed and one unit of $C$ (predator) is produced. The intensity for this event is proportional to the product of the number of $B$ 's and $C$ 's, where $r$ is the constant of proportionality, later referred to as the reaction rate constant. At any time $t$, the waiting time to the next event is exponentially distributed with this intensity.

In a spatial context, prey in one subvolume can escape by moving to another subvolume. If $B_{i}$ and $B_{j}$ denote the population of preys in neighboring subvolumes $i$ and $j$, then

$$
B_{i} \stackrel{q_{i j}}{\longrightarrow} B_{j}
$$

expresses the event that one unit of prey in the $i$ th subvolume moves to the $j$ th. The waiting time for this event is equal to the product of $B_{i}$ and the transport rate constant $q_{i j}$. Depending on the scaling of this constant versus the spatial units, different types of transport may in principle be modeled. In this work we consider the diffusive scaling regime, in which $q_{i j} \propto h^{-2}$, with $h$ a length-scale (e.g., a radius) of the subvolumes. Notably, with a finer discretization (i.e., $h \rightarrow 0$ ), the number of diffusion events will increasingly dominate the Markov chain.

It was Gillespie [17] who popularized simulating independent samples from master equations in general. For RDMEs one of the first practical sampling algorithms was proposed in [12], the Next Subvolume Method (NSM). The NSM is a spatial extension of Gillespie's Direct Method, incorporating features of the Next Reaction Method [16], which was the first sampling algorithm using a sorted event queue for efficiency.

In this work we consider a related method, the All Events Method (AEM) [3]. The algorithm generates next event times for each reaction and diffusion in all subvolumes and stores them in an event queue. It proceeds by repeatedly selecting the event with the smallest time from the event queue, processes it by updating the state, and finally updates the event queue by sampling the next time for the event just processed. Also, at this stage, those rates which have changed due to the state update need to be rescaled (see [3] for details).

Being essentially a spatial extension of the Common Reaction Path method [28], the AEM has the benefit of defining a consistent stochastic flow in the sense of dynamical systems. This means that the result from different simulation runs, e.g., with slightly different model parameters, using the same stream of random numbers will be comparable in a path-wise sense. Besides implying a much reduced variance in statistical estimators, this is also required when evaluating the effect of small perturbations or coefficient uncertainties in a path-wise sense (e.g., root-mean-square, see [3]).

Of relevance to the current application, the AEM stores the waiting times for the next instance of each reaction or diffusion event such that it is possible to estimate with reasonable precision when specific events will happen, notably including diffusion events between subvolumes. Another feature of more practical nature is that, by seeding the random number generators in an identical way, the parallel simulations yield identical results independently on the number of LPs, thus ensuring correctness. These features come at a certain cost, however, as the AEM requires to store more entries in the event queue compared to, e.g., the NSM.

\section{PARALLEL IMPLEMENTATION}

In this section we detail our parallelization of the All Events Method (PAEM), which implements the DLTWE for a general class of RDME models.

In our parallel simulator, the subvolumes of the simulation model are partitioned into subdomains, each of which is assigned to an LP. Each LP evolves the state of its subdomain while maintaining three main data structures: (i) the $s u b$ domain state, i.e., for each subvolume, the number of entities 
of each species (the copy number) as well as the timestamp of the last event affecting the subvolume, (ii) a time-sorted event queue, containing the next occurrence of each event type (reaction or diffusion) for each subvolume in its subdomain, and (iii) a rollback history, which is a time-sorted sequence of events already processed by this LP.

Each LP advances the simulation by processing events that affect its subdomain. The LP repeatedly finds the next event for processing, either in its event queue or in a message from another LP, and processes it by (i) updating the states of affected subvolumes, (ii) adding the event to its rollback history, and (iii) adding the next event of the same type and subvolume to its event queue. If the event is a diffusion event which crosses a subdomain boundary, then a message is transmitted to the neighboring LP; each pair of LPs is connected by a FIFO channel in each direction.

Whenever an LP receives a diffusion message that causes a causality violation (i.e., it is a straggler), it must perform a rollback to the time immediately before the straggler's timestamp, using its rollback history. We have implemented two different versions of the rollback operation: a more costly simple rollback and a less costly selective rollback. The selective rollback is described further below. In the simple rollback, the local time of the LP is reset to the time immediately preceding the timestamp of the straggler, and the events in the rollback history that occur after this timestamp are processed "backwards". All diffusion messages that had been sent by the LP during the rollback interval must be undone by sending corresponding anti-messages to the corresponding LPs. An anti-message cancels any event that was sent earlier with the same or a later timestamp. The receipt of an anti-message triggers rollbacks at the receiving LPs if it cancels a message that has already been processed.

Since rollbacks triggered by stragglers hurt performance, an LP should try not to advance its local simulation time past the timestamp of any diffusion message that will be received in the future. For this purpose, we have developed the DLTWE (Dynamic Local Time Window Estimate) technique, whereby each LP communicates to a subset of its neighboring LPs an estimate of the timestamp of the next diffusion to respective LP; these estimates are obtained from the current contents of the event queue. An LP does not advance its local simulation past the time of the earliest incoming time estimate. The optimism of the simulation is controlled by the tuning of the DLTWE computation, as described in more detail in Section 5.4.

To reduce the impact of rollbacks, we have developed a technique for selective rollback. An LP that receives a straggler or an anti-message performs a refined analysis before executing a rollback. Rather than merely comparing the timestamp of the incoming diffusion message with its local simulation time, the receiving LP finds the causality violations that are incurred by the incoming message. The LP finds the processed events that are causally dependent on the straggler or anti-message using the trace. Only these events are rolled back. The cost of selective rollback is typically significantly lower than the cost of simple rollback.

\subsection{The simulator main loop}

Algorithm 1 is a pseudocode description of the main loop executed by each LP. Lines 2 through 6 define the main data structures. These are

- EventQueue is a time-sorted priority queue containing the scheduled local events;
- SubvolumeState represents the state of each subvolume in the subdomain, i.e., the number of entities of each species in each subvolume, as well as the timestamp of the last event affecting the subvolume,

- History is a time-sorted sequence of events already processed by the LP; old events are regularly removed from the history by fossil collection, which we do not further describe here,

- Channels contains an incoming message channel for each neighboring LP, and

- Dltwe ${ }_{j, i}$ consists of a DLTWE estimate from $\mathrm{LP}_{i}$ to $\mathrm{LP}_{j}$, defined for each pair of neighboring LPs.

For an event $e$, we let e.time denote its timestamp; for a diffusion event $e$, we let $e$.dest denote its destination subvolume. For a subvolume $s$, we let $\operatorname{dom}(s)$ denote the index of the LP to which $s$ belongs.

The main simulator loop consists of two phases. The first phase (lines 8-18), finds the next event to be processed, as follows. First, for each incoming channel, the first message that is not canceled by a later anti-message in the channel, is retrieved by means of the function RETRIEVEMsG. Intuitively, the retrieved message is the first one in the channel that should be processed after all rollbacks induced by anti-messages have been performed. The earliest of these messages is assigned to $e_{\mathrm{msg}}$. If $e_{\mathrm{msg}}$ is a straggler which violates causality in its destination subvolume (checked at line 9) then a rollback is performed. Second, the earliest event $e_{\text {local }}$ in the event queue is read. If $e_{\mathrm{msg}}$ is earlier than $e_{\text {local }}$ (line 12), then $e_{\mathrm{msg}}$ is assigned to $e$ for processing. Otherwise, the event $e_{\text {local }}$ is assigned to $e$ for processing, but only if no DLTWE estimate is violated (line 15). If a DLTWE estimate is violated, the LP blocks until the expected message or a message from another neighbor is received, at which time the main loop is restarted, in order to process the message (line 17 ).

The second phase (lines 19-30) updates the subdomain state of the LP by processing the event $e$ that was selected in the first phase. It starts by checking whether $e$ is a "local straggler", i.e., a local diffusion event (between subvolumes of the same LP) that would cause a local causality error (line 19), in which case a rollback is necessary. Thereafter, $e$ is processed by adding it to the event history (line 21), updating the states of affected subvolumes, and updating the times of future events in the event queue that are affected by the state change(s) (lines 22 through 26). If $e$ is a diffusion to another subdomain, a message is sent (line 28) to the appropriate LP. After that, the DLTWEs are updated (line 30) to inform the neighboring LPs of the estimated times of the next diffusion events.

DLTWEs are computed based on outgoing diffusion events that can be found in a prefix of the event queue, thus not considering diffusion events that are scheduled far into the future. If no relevant diffusion events for a specific LP are found in the prefix, the corresponding DLTWE is set to infinity. The length of the considered prefix is a tuneable parameter of our approach. A short prefix induces less effort for DLTWE computation, but will generate DLTWE estimates for only a subset of neighboring LPs, inducing more optimism in the simulation; too much optimism may result in high cost for rollbacks. A long prefix, on the other hand, costs more effort for DLTWE computation, and will avoid excessive cost of rollbacks, but may in some context also in- 
Algorithm 1: Main loop of Parallel AEM Simulator, executed by each LP.

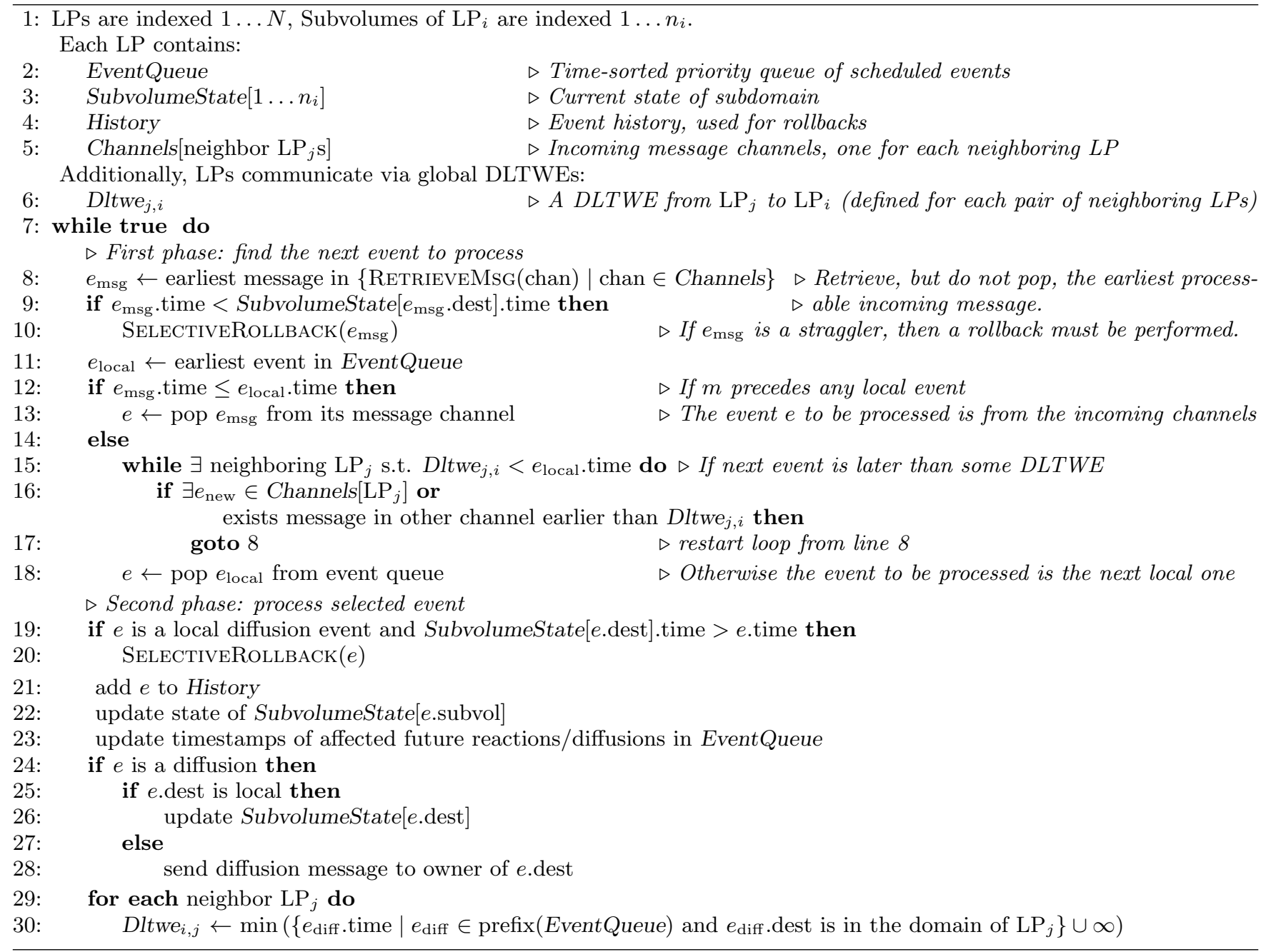

duce too little optimism. How to tune the prefix length to make this trade-off is examined in Section 5.4.

As an optimization, the updates of the DLTWEs at line 30 are performed only when necessary, i.e., when the estimated time of some future inter-LP diffusion event is updated. The DLTWE estimates are communicated using a single memory cell per direction and neighboring LP-pair, which is only written to when this results in a new value, to avoid unnecessary coherence traffic.

\subsection{The SelectiveRollback function}

The function SelectiveRollback $\left(e_{\text {cause }}\right)$, shown in Algorithm 2, reverses the effect of all events processed by an LP at or after time $e_{\text {cause }}$.time, that are causally dependent

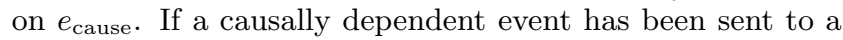
neighbor, a corresponding anti-message will be sent. Different subvolumes may be rolled back to a different timestamp. First, we let $H$ contain the part of the history that may be rolled back (line 2). After that we define a set $D$ of subvolume timestamp pairs, 〈subvolume, timestamp〉. Each pair $\langle s, t\rangle$ defines the time $t$ to which subvolume $s$ has to be rolled back. Initially, if $e_{\text {cause }}$ is a straggler, $D$ must at least contain $\left\langle e_{\text {cause }}\right.$.dest, $e_{\text {cause. }}$.time $\rangle$ (line 4$)$. If $e_{\text {cause }}$ is an anti-message,
$D$ instead contains all destination subvolumes of messages received from $\operatorname{dom}\left(e_{\text {cause }}\right)$ after time $e_{\text {cause }}$.time, and their respective time (line 6 ). Thereafter, $D$ is completed to contain all $\langle$ subvolume, timestamp $\rangle$ pairs that are causally dependent on $e_{\text {cause }}$. More precisely, for every diffusion event between any two subvolumes $s, s^{\prime}$ of this LP at time $t^{\prime \prime}$, we have that if $\langle s, t\rangle \in D$ and $t\left\langle t^{\prime \prime}\right.$, there exists $\left\langle s^{\prime}, t^{\prime}\right\rangle \in D$ such that $t^{\prime} \leq t^{\prime \prime}$ (line 7 ). In the main while loop, the subset of the history $H$ is traversed backwards in time, event by event (line 8 ). Each event being incident on some subvolume occurring in $D$, after the corresponding time $t$, is rolled back. An event $e$ is reverted by reversing the state changes of the affected subvolumes, and updating the intensity of the corresponding reaction or diffusion accordingly. If the rollback was initiated by an anti-message, and $e$ is an incoming diffusion originating from a neighbor LP that did not send this anti-message, then $e$ will be pushed back to the top of its originating message channel (checked at line 12). If one or more diffusion events have been sent to a neighbor $\mathrm{LP}_{j}$ during the rollback interval, a single anti-message will be sent, containing the timestamp of the earliest message sent to $\mathrm{LP}_{j}$ after $t$ (starting at line 14). 
Algorithm 2: Rollback events at or after time $t$.

\begin{tabular}{|c|c|}
\hline & nction SELECTIVEROLLBACK(event $\left.e_{\text {cause }}\right)$ \\
\hline 2: & $H \leftarrow\left\{e \mid e \in\right.$ History and $e$.time $\geq e_{\text {cause }}$.time $\}$ \\
\hline 3: & if $e_{\text {cause }}$ is straggler then \\
\hline 4: & $D \leftarrow\left\{\left\langle e_{\text {cause }} \cdot\right.\right.$ dest,$e_{\text {cause. }} \cdot$ time $\left.\rangle\right\}$ \\
\hline 5: & else $\quad \triangleright e_{\text {cause }}$ is an anti-message \\
\hline 6: & $\begin{aligned} D \leftarrow\{\langle e . \text { dest }, e . \text { time }\rangle \mid & e \in H, \operatorname{dom}(e . \text { subvol })= \\
& \left.\operatorname{dom}\left(e_{\text {cause }} . \text { subvol }\right)\right\}\end{aligned}$ \\
\hline 7: & $D \leftarrow$ extend $D$ under causal dependence \\
\hline 8: & while $e \leftarrow$ pop latest event in $H$ do \\
\hline 9: & if $\exists\langle s, t\rangle \in D$ s.t. \\
\hline ): & $\begin{array}{l}\text { e.subvol }=s \text { and } e . \text { time } \geq t \text { then } \\
\text { revert } e\end{array}$ \\
\hline 11: & Pop $e$ from History \\
\hline 12: & $\begin{array}{c}\text { if } e . \operatorname{subvol} \in \mathrm{LP}_{j} \text { and not (isanti }\left(e_{\text {cause }}\right) \text { and } \\
\left.\operatorname{dom}\left(e_{\text {cause }} \cdot \text { subvol }\right)=\mathrm{LP}_{j}\right) \text { then } \\
\text { push } e \text { back to front of Channels }\left[\mathrm{LP}_{j}\right]\end{array}$ \\
\hline 14: & for each neighbor $\mathrm{LP}_{j}$ do $\triangleright$ send anti-messages \\
\hline 15: & $\begin{array}{l}e_{\text {diff }} \leftarrow \text { earliest rolled back diffusion to } \mathrm{LP}_{j} \\
\text { send anti-message with time } e_{\text {diff.time to } \mathrm{LP}_{j}}\end{array}$ \\
\hline
\end{tabular}

\subsection{The RetrieveMsg function}

The function RETRIEVEMsG(chan) returns the first message in the incoming channel chan that can be meaningfully processed, i.e., it is not undone by a corresponding antimessage already present in chan. The function starts by finding the timestamp of the earliest anti-message in the channel (line 3). Thereafter, messages are popped and discarded from the channel, until either the first message preceding the earliest anti-message is encountered, or until the anti-message itself is encountered (lines 4-7). In the former case, the function returns the message immediately without popping it from chan. In the latter case, a rollback corresponding to the anti-message is performed (line 8) and the procedure is repeated. Thus, there are two possible states of the channel after the completion of the function: $a$ ) either there are no anti-messages left in the channel, or $b$ ) the first message in the channel is a diffusion event and has a time earlier than the time of the earliest anti-message in the channel.

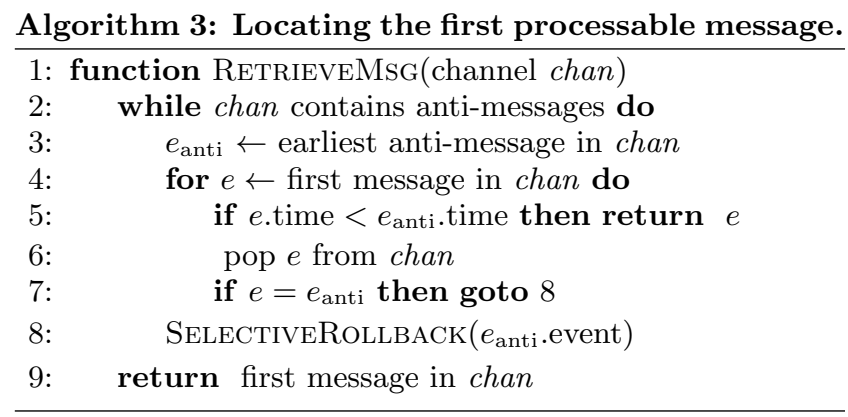

\section{PERFORMANCE EVALUATION}

In this section, we evaluate the performance of our parallelization technique. The aim is to answer the following questions.
- How does our technique scale with the number of LPs? In Section 5.2 we determine the speedup obtained on benchmarks, and investigate the dependency on model parameters.

- How does the parallelized simulator behave? In Section 5.3 we describe how the computation effort is spent on different activities, exposing potential bottlenecks.

- How should the DLTWE technique be tuned? In Section 5.4 we describe how to tune the cost for the computation of the DLTWE estimate against the gain in reduced rollback frequency.

- How does the DLTWE technique compare to other techniques? In Section 5.5, we compare the DLTWE technique to adaptive optimism control techniques, that are based on local history. In Section 5.6, we compare our parallel simulator to other existing simulators for RDME models.

The performance was evaluated on three sets of benchmarks, described in more detail in Section 5.1. All experiments were run on a 4-socket Intel Sandy Bridge E5-4650 machine, each socket having 8 cores and a $20 \mathrm{MB}$ shared L3-cache. Hyperthreading was used, resulting in a total of 16 hardware threads per processor. An LP is always assigned to a single thread. Speedup is defined as the wall-clock time of the sequential algorithm (AEM) over the wall-clock time of parallel algorithm (PAEM). Three-dimensional geometries were constructed using Comsol Multiphysics 4.3 and converted to computational models using the URDME framework [11]. The two-dimensional structured meshes used in the spatial predator and prey model were constructed using custom Matlab scripts. The resulting meshes were divided into subdomains using the multilevel k-way partitioning method provided by the Metis library [22]. Metis optimizes the partitioning for minimal number of diffusions crossing subdomain boundaries, while maintaining an equal number of subvolumes in each subdomain. The partitioning provides a balanced simulator workload, which we believe has an impact on the parallel performance.

\subsection{Benchmarks}

We investigated the behavior of our simulator on three benchmarks. In the first benchmark we evaluated the scaling as a function of the geometry and the ratio of diffusion to reaction events (D:R). The $\mathrm{D}: \mathrm{R}$ was measured during a sequential profiling run. The second benchmark is a spatial predator and prey model in two dimensions, which was previously used for performance evaluation by others [34]. The last benchmark is the simulation of the Min-protein system in a three-dimensional model of the E. Coli bacterium.

\subsubsection{Reversible isomerization}

We created spatial models from different three-dimensional geometries, namely a sphere, a disc, and a rod, all of equal volume. The RDME model considered consists of two freely diffusing species, $A$ and $B$, each with initial copy numbers of 1000 per subvolume. We prescribed the simplest possible reversible isomerization

$$
A \stackrel{c}{\rightarrow} B, \quad B \stackrel{c}{\rightarrow} A
$$

where the reaction rate $c$ is selected such that the D:R is 1 when both species diffuse at a diffusion rate of 1 . The diffusion rates of both species were varied in $\{1,100\}$, thereby 
increasing the D:R. We also varied the volume of the geometries in $\{1,10,100\}$. For the sphere and the disc we did this by increasing the radius, keeping the height of the disc at the constant value 0.2 . For the rod, the radius was kept at the value 0.2 while the length was increased. As all discretization parameters remained the same for all model configurations, the number of subvolumes in each model grew to approximately $\{1500,15000,150000\}$. In the following, we refer to the specific model configurations as $[\mathrm{v} x, \mathrm{~d} y]$, denoting that the model has a volume of $x$ and that the diffusion rates for both species are $y$.

\subsubsection{Spatial predator-prey model}

This benchmark is the spatial extension of the LotkaVolterra model, proposed by Schinazi [30]. We use the model parameters proposed by Wang et al. [34]. The system contains three species, $A, B$, and $C$, where the initial copy number for each is set to 1000 per subvolume. The model reads

$$
\begin{aligned}
A+B & \stackrel{0.01}{\longrightarrow} A+2 B, \\
B+C & \stackrel{0.01}{\longrightarrow}, 2 C \\
C & \stackrel{1}{\rightarrow} \emptyset .
\end{aligned}
$$

Note that the count of species $A$ is not changed in the first reaction, but $A$ is a factor of the total reaction intensity. The geometry is a two-dimensional square with a varying side of length $\{64,200,400\}$ units and with square subvolumes of unit area. The diffusion rates of species $B$ and $C$ are either $d_{B}^{1}=2.5$ and $d_{C}^{1}=5$, or $d_{B}^{2}=5$ and $d_{C}^{2}=10$, while $d_{A}=0$ in all cases. In the first case, the D:R is approximately 1 , and about 2 in the second.

\subsubsection{A model of the Min-protein system}

As a rather challenging benchmark we used a model of a Min-protein system in a three-dimensional model of an E. Coli bacterium [13]. The model contains five chemical species interacting in a system of five reactions. The geometry is pill-shaped, resulting from the union of a cylinder with two spheres (Figure 1). The complete set of reaction- and diffusion-rates can be found in [13], and the model is also available for download in the current release of URDME [2]. We simulated the model at two different mesh resolutions, hence at two different ratios of reaction to diffusion events since the diffusion rate is inversely proportional to the square of the subvolume length. The coarse mesh (Figure 1A) contained 1555 subvolumes and the D:R was approximately 250 . In the fine mesh (Figure 1B) the system consisted of 13307 subvolumes, and the D:R was about 1400 .

\subsection{Scalability}

In this section, we evaluate how the simulator performance scales with increasing LP-count, and how the scaling depends on the particular model. In order to relate the models better to the measured performance we identify four potential performance indicators:

- Subvolume count: The number of subvolumes in the model.

- The diffusion to reaction ratio (D:R): The ratio of simulated diffusion to reaction events.

- Average degree: The average number of neighbors of each LP.

- Inter-LP diffusion ratio (Inter-LPD): The number of diffusions crossing subdomain boundaries over the total number of diffusions. We also tried including the
A
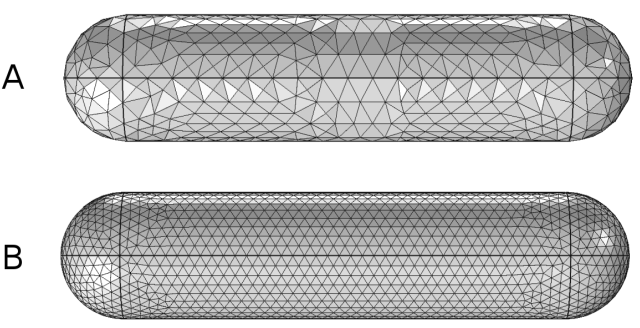

Figure 1: The spatial discretization of the E.coli bacterium geometry; coarse-grained (A) and finegrained (B) tetrahedral meshes.

reactions into the ratio, but this yielded a worse indicator. We discuss the impact of reactions separately under the D:R.

In Table 1 we present an overview of the benchmark configurations together with the introduced indicators. As the indicators inter-LP diffusion ratio and the average degree depend on the number of partitions, the values are listed for the partitioning to 16,32 and 64 subdomains. We also list the sequential and parallel wall-clock time measured for all model configurations. Note that to measure the sequential time we used the sequential version of the algorithm (AEM), thus no parallelization overhead is included in the measurement. Moreover, the simulated time range was freely varied for each configuration, thus no direct relationship exists between wall-clock times shown in different rows.

Lastly, we list the parallel efficiency for all experiments and the same set of partitions. The parallel efficiency calculates as $T_{1}\left(T_{N} * N\right)^{-1}$, where $T_{1}$ is the sequential simulation time and $T_{N}$ the parallel simulation time using $N$ LPs.

We investigated the relationship of the introduced indicators to the measured parallel efficiency. To study the influence of the inter-LP diffusion ratio (inter-LPD) we observe the scaling of the rod, disc and sphere models at the $[\mathrm{d} 1]$ configuration shown in Figures $2 \mathrm{a}, 2 \mathrm{~b}$ and $2 \mathrm{c}$. We see that large models (v100) scale significantly better than models of medium (v10) and small size (v1). As shown in Table 1, a large model size leads to a high private work-load per LP and thus a low inter-LPD. Furthermore, large models with a lower inter-LPD (e.g., rod) achieve a higher parallel efficiency than models with a higher inter-LPD (e.g., sphere). The inter-LPD increases at an increasing LP-count, as the average subdomain size and the number of internal diffusions decreases. This noticeably affects the scaling of small models (v1), where doubling the LP-count significantly reduces the parallel efficiency. Hence, we find that the inter-LPD is an accurate indicator for the parallel performance of our simulator.

To study the impact of an increasing diffusion to reaction ratio ( $\mathrm{D}: \mathrm{R})$ we present the scaling of the sphere model at configurations [d1] and [d100] shown in Figure 4. Here we find that the difference in parallel performance due to the increased D:R is small $(<10 \%)$. Furthermore, for large models (v100) we observe that the parallel performance is independent of the D:R, as shown in Table 1. This is an unexpected finding, as we assumed that the D:R has a stronger influence on the scaling due to its effect on private workload.

To study how the parallel efficiency depends on the average degree in isolation, we compare different configurations 


\begin{tabular}{|c|c|c|c|c|c|c|c|c|c|c|c|c|c|c|c|c|}
\hline \multirow[t]{2}{*}{ Model } & \multirow[t]{2}{*}{ Conf. } & \multirow[t]{2}{*}{ \#Subvol. } & \multirow[t]{2}{*}{$\mathrm{D}: \mathrm{R}$} & \multicolumn{3}{|c|}{ Avg. Degree } & \multicolumn{3}{|c|}{ Inter-LPD. \% } & \multicolumn{4}{|c|}{ Time $[\mathrm{s}]$} & \multicolumn{3}{|c|}{ Efficiency } \\
\hline & & & & 16 & 32 & 64 & 16 & 32 & 64 & Seq. & 16 & 32 & 64 & 16 & 32 & 64 \\
\hline \multirow{6}{*}{ Sphere } & {$[\mathrm{v} 1, \mathrm{~d} 1]$} & 1437 & 1 & 7.6 & 8.8 & 10.3 & 24 & 32 & 42 & 198.7 & 34.7 & 29.9 & 21.9 & 0.36 & 0.21 & 0.14 \\
\hline & {$[\mathrm{v} 1, \mathrm{~d} 100]$} & 1437 & 105 & 7.6 & 8.8 & 10.3 & 24 & 32 & 42 & 216.4 & 45.5 & 40.5 & 37.9 & 0.3 & 0.17 & 0.09 \\
\hline & {$[\mathrm{v} 10, \mathrm{~d} 1]$} & 13575 & 1 & 7.8 & 8.8 & 10.8 & 12 & 15 & 21 & 259.8 & 37.7 & 23.1 & 13.7 & 0.43 & 0.35 & 0.3 \\
\hline & {$[\mathrm{v} 10, \mathrm{~d} 100]$} & 13575 & 107 & 7.8 & 8.8 & 10.8 & 12 & 15 & 21 & 293.7 & 43.3 & 28.2 & 17.1 & 0.42 & 0.33 & 0.27 \\
\hline & {$[\mathrm{v} 100, \mathrm{~d} 1]$} & 135228 & 1 & 7.9 & 9.8 & 10.8 & 6 & 8 & 10 & 545.1 & 64.1 & 31.1 & 12.6 & 0.53 & 0.55 & 0.68 \\
\hline & {$[\mathrm{v} 100, \mathrm{~d} 100]$} & 135228 & 109 & 7.9 & 9.8 & 10.8 & 6 & 8 & 10 & 476.1 & 53.3 & 24.5 & 11.6 & 0.56 & 0.61 & 0.64 \\
\hline \multirow{6}{*}{ Disc } & {$[\mathrm{v} 1, \mathrm{~d} 1]$} & 1555 & 1 & 4.1 & 5 & 5.7 & 15 & 22 & 33 & 186.9 & 31.3 & 23.7 & 23.1 & 0.37 & 0.25 & 0.13 \\
\hline & {$[\mathrm{v} 1, \mathrm{~d} 100]$} & 1555 & 91 & 4.1 & 5 & 5.7 & 15 & 22 & 33 & 190.2 & 33.5 & 26.8 & 30 & 0.36 & 0.22 & 0.1 \\
\hline & {$[\mathrm{v} 10, \mathrm{~d} 1]$} & 13452 & 1 & 4.4 & 4.7 & 5.2 & 5 & 8 & 11 & 203.4 & 27.3 & 14.9 & 8.8 & 0.47 & 0.43 & 0.36 \\
\hline & {$[\mathrm{v} 10, \mathrm{~d} 100]$} & 13452 & 85 & 4.4 & 4.7 & 5.2 & 5 & 8 & 11 & 204.4 & 26.8 & 15.9 & 9.7 & 0.48 & 0.4 & 0.33 \\
\hline & {$[\mathrm{v} 100, \mathrm{~d} 1]$} & 125537 & 1 & 4.2 & 4.6 & 5.1 & 2 & 3 & 4 & 376.9 & 45.7 & 20.1 & 7.4 & 0.52 & 0.59 & 0.8 \\
\hline & {$[\mathrm{v} 100, \mathrm{~d} 100]$} & 125537 & 82 & 4.2 & 4.6 & 5.1 & 2 & 3 & 4 & 282.7 & 34.2 & 14.9 & 5.5 & 0.52 & 0.59 & 0.8 \\
\hline \multirow{6}{*}{ Rod } & {$[\mathrm{v} 1, \mathrm{~d} 1]$} & 1429 & 1 & 1.9 & 1.9 & 2.8 & 13 & 27 & 54 & 174.7 & 27.1 & 22.9 & 27.1 & 0.4 & 0.24 & 0.1 \\
\hline & {$[\mathrm{v} 1, \mathrm{~d} 100]$} & 1429 & 90 & 1.9 & 1.9 & 2.8 & 13 & 27 & 54 & 177.6 & 31.1 & 30.1 & 33.8 & 0.36 & 0.19 & 0.08 \\
\hline & {$[\mathrm{v} 10, \mathrm{~d} 1]$} & 14000 & 1 & 1.9 & 1.9 & 2 & 1 & 2 & 5 & 224.2 & 28 & 14.7 & 8.3 & 0.5 & 0.48 & 0.42 \\
\hline & {$[\mathrm{v} 10, \mathrm{~d} 100]$} & 14000 & 90 & 1.9 & 1.9 & 2 & 1 & 2 & 5 & 232.6 & 27.7 & 15.6 & 9.3 & 0.53 & 0.47 & 0.39 \\
\hline & {$[\mathrm{v} 100, \mathrm{~d} 1]$} & 139139 & 1 & 1.9 & 1.9 & 2 & 0 & 0 & 0 & 325.5 & 40.1 & 18 & 6.4 & 0.51 & 0.56 & 0.79 \\
\hline & {$[\mathrm{v} 100, \mathrm{~d} 100]$} & 139139 & 91 & 1.9 & 1.9 & 2 & 0 & 0 & 0 & 357 & 42.2 & 19.7 & 6.9 & 0.53 & 0.57 & 0.81 \\
\hline \multirow{6}{*}{$\begin{array}{l}\text { Pred.- } \\
\text { Prey }\end{array}$} & {$[\mathrm{n} 64, \mathrm{~d} 1]$} & 4096 & 1 & 4.1 & 4.7 & 4.9 & 6 & 10 & 14 & 203.5 & 29.2 & 22.8 & 12.2 & 0.44 & 0.28 & 0.26 \\
\hline & {$[\mathrm{n} 64, \mathrm{~d} 2]$} & 4096 & 2 & 4.1 & 4.7 & 4.9 & 6 & 10 & 14 & 324.6 & 51.6 & 41.2 & 33.4 & 0.39 & 0.25 & 0.15 \\
\hline & {$[\mathrm{n} 200, \mathrm{~d} 1]$} & 40000 & 1 & 4.1 & 4.6 & 5 & 2 & 3 & 5 & 371.2 & 44.2 & 22.7 & 11 & 0.53 & 0.51 & 0.53 \\
\hline & {$[\mathrm{n} 200, \mathrm{~d} 2]$} & 40000 & 2 & 4.1 & 4.6 & 5 & 2 & 3 & 5 & 592.6 & 72.3 & 34.6 & 20.9 & 0.51 & 0.54 & 0.44 \\
\hline & {$[\mathrm{n} 400, \mathrm{~d} 1]$} & 160000 & 1 & 4 & 4.6 & 5.1 & 1 & 2 & 2 & 286.1 & 31.5 & 13.8 & 5.8 & 0.57 & 0.65 & 0.78 \\
\hline & {$[\mathrm{n} 400, \mathrm{~d} 2]$} & 160000 & 2 & 4 & 4.6 & 5.1 & 1 & 2 & 2 & 387.1 & 50.5 & 22.2 & 9.1 & 0.48 & 0.54 & 0.67 \\
\hline Min- & [coarse,--] & 1555 & 304 & 5.2 & 7.5 & 8.7 & 20 & 27 & 38 & 126 & 29.7 & 23 & 24 & 0.27 & 0.17 & 0.08 \\
\hline System & {$[$ fine, -$]$} & 13307 & 1517 & 4.9 & 7.3 & 8.9 & 10 & 14 & 20 & 539.9 & 80.4 & 53.1 & 34.1 & 0.42 & 0.32 & 0.25 \\
\hline
\end{tabular}

Table 1: Overview of benchmark characteristics and results.

of geometries with the same Inter-LPD. Namely, the models disc $[\mathrm{v} 10, \mathrm{~d} 1]$ and sphere $[\mathrm{v} 100, \mathrm{~d} 1]$, both of which have a Inter-LPD of $8 \%$ at the partitioning on 32 LPs. We find that the sphere model has a higher average degree than the disc model and the parallel efficiency is likewise increased. Nonetheless, as the models are of different subvolume sizes, we can not rule out the influence of unknown factors that correlate with the average degree.

Lastly, we observe the effect of the subvolume count indicator. It can be seen in Table 1 that a correlation with the Inter-LPD and thus the parallel efficiency exist. Furthermore, the efficiency for simulation of large models (v100) increases at increasing LP-count, which is not the case for small or medium size models. We suspect that this outcome is attributable to cache effects, as the partitioned model may fit better into core-local cache levels.

To visualize the correlation of the inter-LP diffusion ratio and subvolume count indicators to the parallel efficiency we applied least-squares curve fitting to the data for all [d1] models simulated on 64 LPs, as shown in Table 1. In Figure 3 we see the inter-LPD to parallel efficiency data fitted with a negative exponential function, and the subvolume count to parallel efficiency correlation fitted by a log-linear relationship.

\subsection{Detailed Behavior}

In this section, we study in detail how the effort of the simulator is allocated. The DLTWEs were tuned to achieve
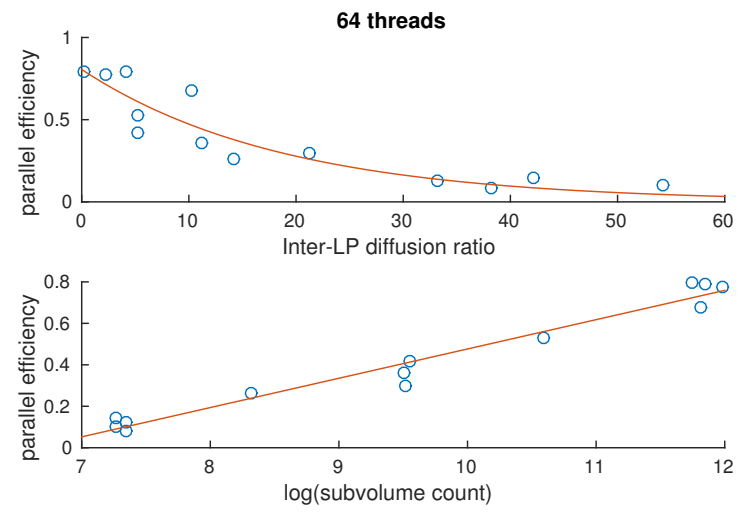

Figure 3: Curve fitting of the inter-LPD and subvolume-count indicators to the parallel efficiency for all [d1] models simulated on 64 LPs.

the best performance for each model; hence the degree of optimism varies, and as a consequence the allocation of effort may be distributed differently. To measure the different parts of the effort, a lightweight instrumentation of the simulator was performed. The instrumentation allows us to break down the execution time into six parts of interest (line numbers refer to Algorithm 1): 


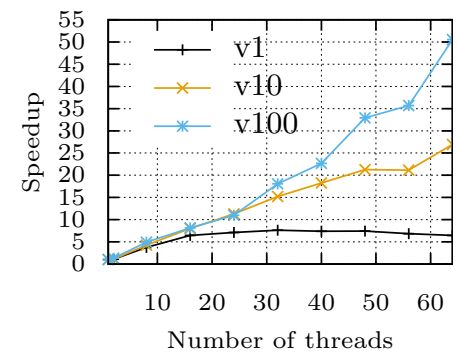

(a) $\operatorname{Rod}[\mathrm{d} 1]$

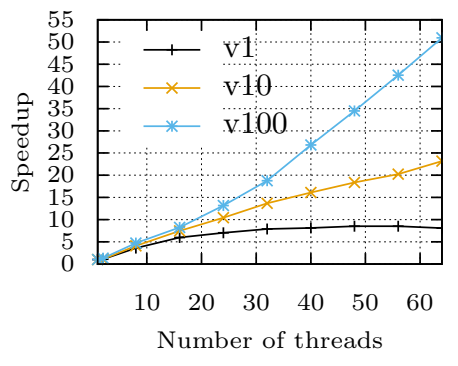

(b) Disc[d1]

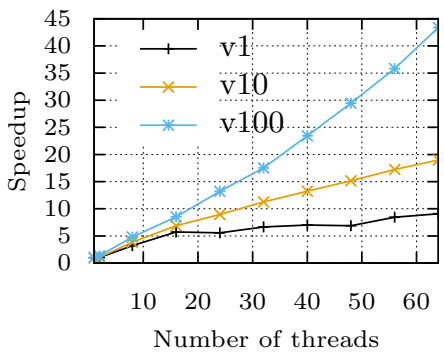

(c) Sphere[d1]

Figure 2: Speedup for different configurations of the geometries rod, disc and sphere, the size is varied.

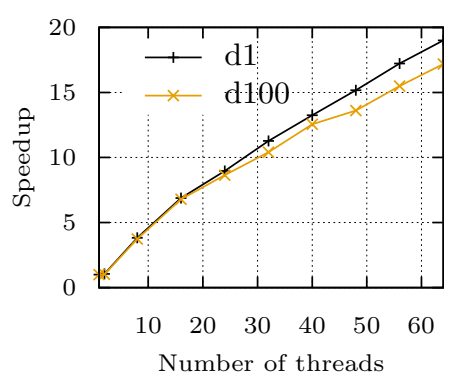

Figure 4: Speedup for the sphere[v10] model, D:R d1 and d100.

- Waiting: Time spent on blocking due to DLTWEs. (lines 15-17)

- Rollbacks: Time spent on processing anti-messages and rollbacks. (lines 8,9 and 20).

- Redo: Time spent on redoing work that has been undone by a preceding rollback. We estimate that the forward processing time is roughly equal to the backward processing time of an event, thus we estimate this value to be the same as Rollbacks.

- Local work: Time spent on processing of local events, other than events that could be attributed to Redo. (roughly lines 21-28, when $e$ originates from the local event $e_{\text {local }}$ at line 18)

- Messaging: Time spent on processing of diffusion messages other than anti-messages and messages that could be attributed to Redo. (roughly lines 21-28, when $e$ originates from the message $m$ at line 13)

- DLTWE computation: Time spent on computing new DLTWEs, including scanning of the event queue. (line 30)

Of the above, Local work and Messaging are considered useful work, and the other parts are referred to as non-work.

The results of the breakdown analysis for the rod[d1], disc[d1] and sphere[d1] models, large size (v100), are shown in Figure 5. We see that the non-work part is completely dominated by blocking due to DLTWEs. Only a lesser part of the time is spent on rollbacks. Hence, the DLTWEs lead to a largely conservative execution for these models. For the sphere model, more time is spent on the processing of messages in relation to the other models. This difference is explained by the increased connectivity of the sphere model,

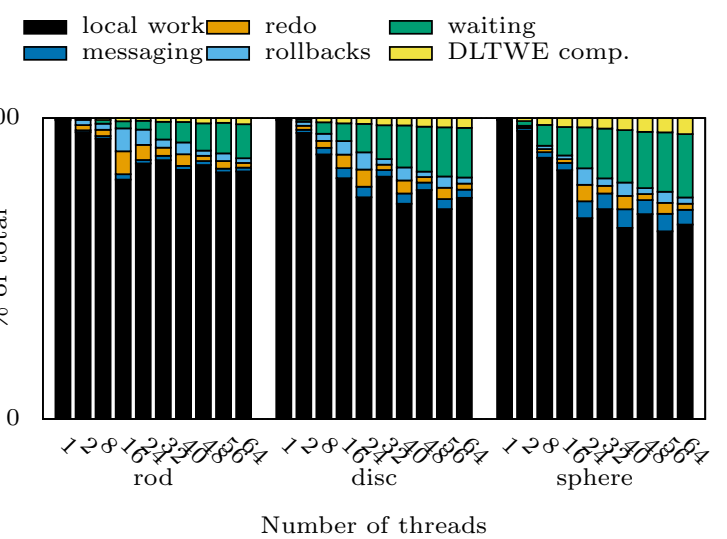

Figure 5: Breakdown of the execution time for the rod, disc and sphere models, size v100 and D:R d1.

whose average degree is the double of that of the disc model, and five-fold in comparison to the rod.
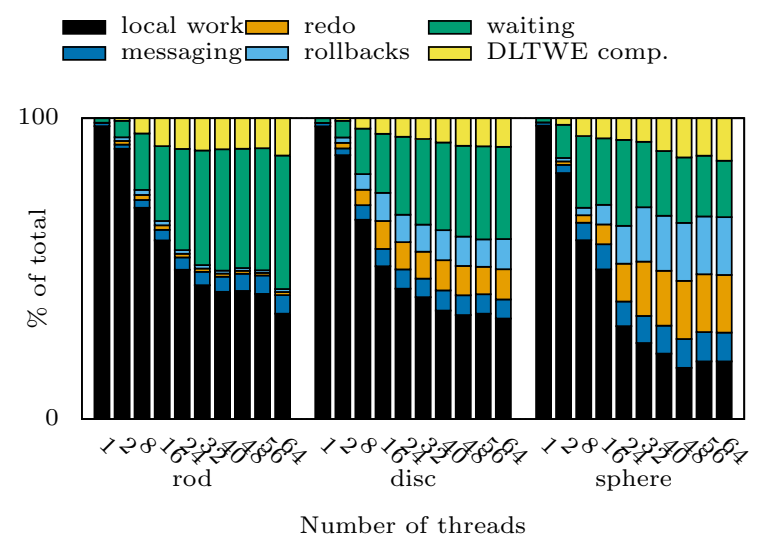

Figure 6: Breakdown of the execution time for the rod, disc and sphere models, size v1 and D:R d1.

In Figure 6, a corresponding breakdown analysis for the small models (v1) is shown. Here we see that a much larger portion of the non-work time is spent on rollbacks, for the disc and the sphere models. The DLTWEs have been tuned for optimal performance in each case. For the small models (v1), we see that for the best performance, a more optimistic (i.e., allowing for more rollbacks) simulation is better. Over- 
all, more time is spent on parallel overhead for small models, which is in line with our expectation, as the amount of private work per LP is very small.

\subsection{Tuning the DLTWE Computation}

In this section, we discuss how to tune the DLTWE computation, and we show how the selective rollback technique affects the performance in comparison to using non-selective rollbacks.

The EventQueue of Algorithm 1 is implemented as two separate queues, one for reactions and one for diffusions. The diffusion queue contains both local and inter-LP diffusion events. The DLTWEs are produced by scanning the events in the diffusion queue (line 30 in Algorithm 1). The length of the prefix being scanned is a tunable parameter of our simulator, that affect the number of neighbors of each LP for which the DLTWEs are updated. Scanning a longer prefix of the diffusion queue results in a greater fraction of the DLTWEs being updated, and thus a more conservative simulation; furthermore, it requires more effort to update the DLTWEs. Scanning a shorter prefix results in fewer DLTWEs being updated (and thus set to infinity), and a more optimistic simulation.

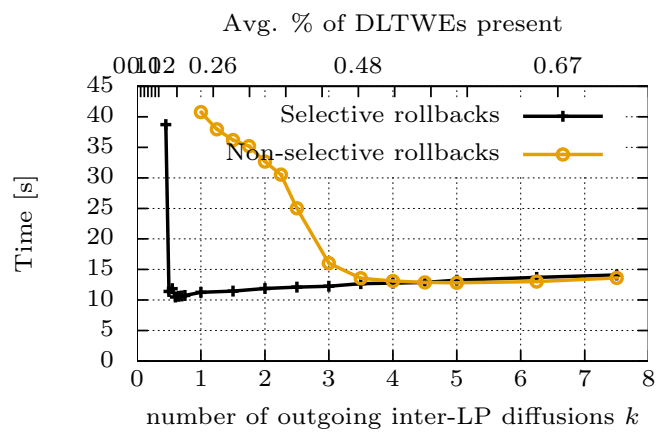

Figure 7: Total execution time for varying number of outgoing inter-LP diffusion events $k$ being scanned, and average percentage of DLTWEs computed on the Predator-Prey[n200,d1] benchmark, on 64 threads.

We analyze how the performance depends on the number of outgoing inter-LP diffusions $k$ found while scanning a prefix of the diffusion queue, and also how this dependency is affected by the total cost of performing rollbacks during a simulation. For this, we run simulations under a range of scan prefix lengths, on the Predator-prey[n200,d1] model, both using the selective rollback technique and using the non-selective rollbacks. The results are displayed in Figure 7. On the $\mathrm{x}$-axis above the plot, the performance is related to the average percentage of DLTWEs being updated. On the $\mathrm{x}$-axis below, the performance is related to the number of outgoing inter-LP diffusions $k$. The execution time of the simulation using selective rollbacks and using non-selective rollbacks are shown for different lengths of the scan prefix (and hence for different $k$ ). For a smaller $k$, the performance of the non-selective rollback starts to decline when the percentage of the DLTWEs being updated passes below $50 \%$. For the selective rollback, the performance does not start to decline until $5 \%$.

We see that in general the selective rollback technique always results in a superior or similar performance in compari- son to the non-selective rollbacks. The optimal $k$ is different for the two techniques, using selective rollbacks it is substantially shorter. This is because the effort of rollbacks is much smaller, and thus the performance improves, as optimism increases, even though the number of stragglers increase. We also see that in general, the best performance is achieved when quite a modest percentage of the DLTWEs between the LPs are known.

\subsection{Comparison to other techniques.}

In this part we compare the DLTWE-synchronization technique to an adaptive protocol guided by the LP's local history, namely the Probabilistic Adaptive Direct Optimism Control (PADOC) proposed by Ferscha [14]. PADOC was implemented in our simulator, replacing the DLTWE synchronization. We have used non-selective rollbacks in this comparison, as it was more efficient when using the PADOC algorithm.

The PADOC algorithm relies on message arrival statistics that are continuously collected on each LP. At each advance of the local simulation time, the LP computes an estimate of the next message arrival time based on the statistics and the last arrival time. Depending on the distance from the current simulation time to the estimate, the LP decides to block for a constant amount of real time or or to proceed with optimistic execution of local events. To be exact, the decision is made by sampling of a sigmoidal probability density function described by a mean at the estimated future arrival time. The steepness of the probability distribution function is scaled with a constant in the range [0 1$]$, where a value closer to 1 implies a stronger confidence in the estimator. In our experiments PADOC obtained the best performance at a scaling constant of 0.1 . This suggest a large variance of the message arrival times in the simulations. We used the arithmetic mean as the estimator of message arrival statistics.

We evaluated PADOC on two benchmarks; the spatial predator and prey model at the [n400,d1] configuration, and the Min-system at the [fine] configuration. The speedup for both models simulated using the PADOC or DLTWE protocol is shown in Figure 8a, 8c. For both models, the DLTWE outperforms PADOC by a large margin. The breakdown of the execution time is shown in Figure $8 \mathrm{~b}$ and $8 \mathrm{~d}$. For each LP-count, the execution time is normalized to the DLTWE time, the left bar. We see that in general, DLTWE keeps the time spent on rollbacks at a very modest level. It should be noted that in the breakdown figures, the relative portion of the waiting time is slightly bigger for the DLTWE than if selective rollbacks would have been used. For PADOC, waiting for neighbors and performing rollbacks takes up a greater part of the total execution time. As the number of LPs increases, the failure to accurately predict arrivals of messages carries an increasingly significant cost.

\subsection{Relation to other works}

In this section we discuss the performance of our simulator using the DLTWE technique in relation to other works. We would like to point out that is difficult to make a fair comparison to other approaches, as previously used methods differ from our approach (e.g., other numerical algorithms or distributed instead of shared-memory). The single previously published RDME benchmark that can be found in the literature for the amount of LPs considered by us was 


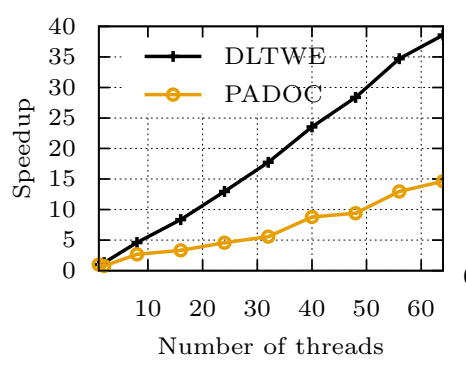

(a) Speedup Predator-Prey

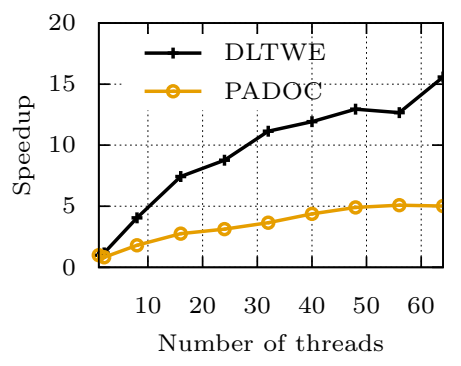

(c) Speedup Min-System

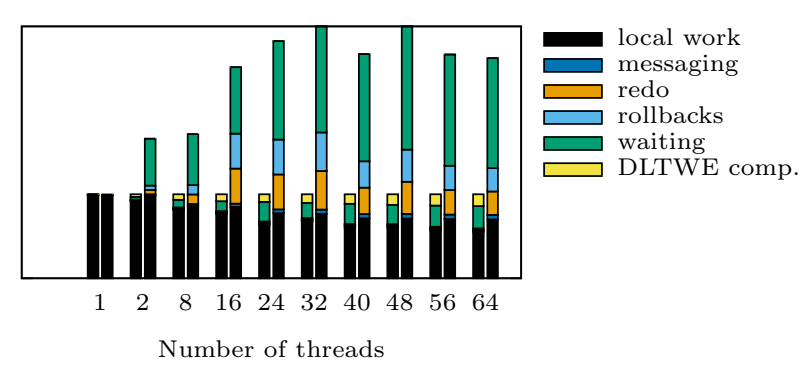

(b) Breakdown Predator-Prey

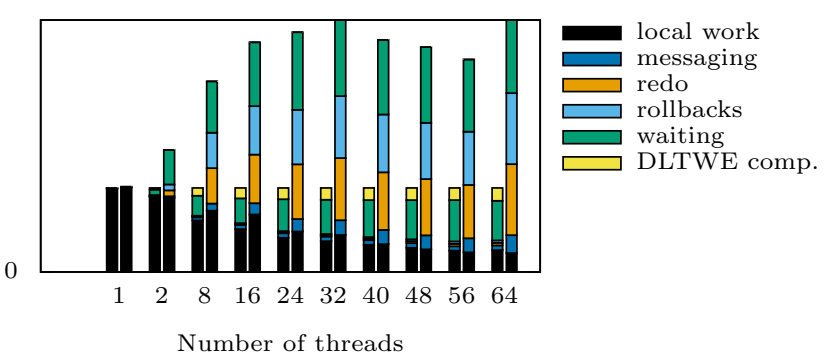

(d) Breakdown Min-System

Figure 8: Comparison of DLTWE and PADOC on the Predator-Prey[n400,d1] and Min-System[fine] models. In (a) and (c) the speedup of the DLTWE and the PADOC method is shown. In (b) and (d) a breakdown of how the time is spent is shown.

the spatial predator and prey model presented by Wang et al. [34]. The parallel simulator used in the study is the Abstract Next Subvolume Method (ANSM), a distributedmemory implementation of the NSM using the Breathing Time-Warp protocol for synchronization. Our speedup and the ANSM speedup taken from [34], Figure 2b, are shown in Table 2.

\begin{tabular}{lrrrr} 
Simulator (Protocol) & 8 LPs & 16 LPs & 32 LPs & 64 LPs \\
\hline ANSM (BTW) & $4 \mathrm{x}$ & $6 \mathrm{x}$ & $11 \mathrm{x}$ & $20 \mathrm{x}$ \\
PAEM (DLTWE) & $4.5 \mathrm{x}$ & $8.4 \mathrm{x}$ & $16.4 \mathrm{x}$ & $33.9 \mathrm{x}$
\end{tabular}

Table 2: Speedups obtained on the spatial predator prey model on a $200 \times 200$ grid using the ANSM and PAEM simulators.

\section{CONCLUSION}

We have presented a new technique for inter-LP synchronization in PDES. It is designed to be suitable when simulating models in which the time intervals between successive inter-LP events are highly variable and have no lower bounds, as in the spatial stochastic simulation that we have considered. Our DLTWE technique enables a detailed control of the amount of optimism in the simulation, which can be tuned to achieve desired accuracy of information communicated between LPs. We have shown how using a technique for selective rollbacks, the cost of optimism decreases, thus making it beneficial to allow for more optimism in the simulation.
With our implementation we have shown that the DLTWE technique is well suited to the setting of spatial stochastic simulations, and that it performs well on realistic problems in a shared memory environment. Notably, the DLTWE enables a parallel scaling which compares favorably to other inter-LP synchronization techniques described in the literature, as well as other parallelization efforts that have been reported in the literature.

\section{ACKNOWLEDGMENTS}

This work was supported in part by the Swedish Foundation for Strategic Research through the CoDeR-MP project as well as the Swedish Research Council within the UPMARC Linnaeus centre of Excellence.

\section{REFERENCES}

[1] R. Ayani and H. Rajaei. Parallel simulation using conservative time windows. In Proceedings of the 24th Winter Simulation Conference, pages 709-717, Arlington, VA, USA, December 13-16 1992. ACM.

[2] P. Bauer, B. Drawert, S. Engblom, and A. Hellander. Urdme v. 1.2: User's manual. Technical Report 2012-036, Uppsala University, 2012.

[3] P. Bauer and S. Engblom. Sensitivity estimation and inverse problems in spatial stochastic models of chemical kinetics. volume 103 of Lecture Notes in Computational Science and Engineering, pages 519-527. Springer Switzerland, 2015. 
[4] D. Bernstein. Simulating mesoscopic reaction-diffusion systems using the gillespie algorithm. Phys. Rev. E, 71(4):041103, 2005.

[5] C. Carothers, D. Bauer, and S. Pearce. ROSS: a high-performance, low memory, modular time warp system. In Proceedings of the 14th Workshop on Parallel and Distributed Simulation, pages 53-60, Bologna, Italy, May 28-31 2000. IEEE.

[6] G. Chen and B. Szymanski. Lookback: a new way of exploiting parallelism in discrete event simulation. In Proceedings of the 16th Workshop on Parallel and Distributed Simulation, pages 138-147, Los Alamitos, CA, USA, May 12-15 2002. IEEE.

[7] L. Chen, Y. Lu, Y. Yao, S. Peng, and L. Wu. A well-balanced time warp system on multi-core environments. In Proceedings of the 25th Workshop on Parallel and Distributed Simulation, pages 1-9, Nice, France, June 14-17 2011. IEEE.

[8] S. H. D. Ball. The adaptive time-warp concurrency control algorithm. In Proceedings of the SCS Multiconference on Distributed Simulation, pages 174-177, San Diego, CA, USA, Jan. 17-19 1990. SCSI.

[9] S. R. Das. Adaptive protocols for parallel discrete event simulation. J. Oper. Res. Soc., 51(4):385-394, 2000 .

[10] L. Dematté and T. Mazza. On parallel stochastic simulation of diffusive systems. volume 5307 of Lecture Notes in Computer Science, pages 191-210. Springer Berlin Heidelberg, 2008.

[11] B. Drawert, S. Engblom, and A. Hellander. URDME: a modular framework for stochastic simulation of reaction-transport processes in complex geometries. BMC Syst. Biol., 6(1):76, 2012.

[12] J. Elf and M. Ehrenberg. Spontaneous separation of bi-stable biochemical systems into spatial domains of opposite phases. Syst. biol., 1(2):230-236, 2004.

[13] D. Fange and J. Elf. Noise-Induced Min Phenotypes in E. coli. PLoS Comput. Biol., 2(6):e80, 2006.

[14] A. Ferscha. Probabilistic adaptive direct optimism control in time warp. In Proceedings of the 9th Workshop on Parallel and Distributed Simulation, pages 120-129, Lake Placid, NY, USA, June 13-16 1995. IEEE.

[15] R. M. Fujimoto. Parallel discrete event simulation. Comm. of the ACM, 33(10):30-53, 1990.

[16] M. A. Gibson and J. Bruck. Efficient exact stochastic simulation of chemical systems with many species and many channels. J. Phys. Chem. A, 104(9):1876-1889, 2000.

[17] D. T. Gillespie. Exact stochastic simulation of coupled chemical reactions. J. Phys. Chem., 81(25):2340-2361, 1977.

[18] S. Jafer, Q. Liu, and G. A. Wainer. Synchronization methods in parallel and distributed discrete-event simulation. Simul. Model. Pract. Th., 30:54-73, 2013.

[19] S. Jafer and G. Wainer. Conservative vs. optimistic parallel simulation of DEVS and cell-DEVS: A comparative study. In Proceedings of the 2010 Summer Computer Simulation Conference, pages 342-349, Ottawa, Canada, July 11-14 2010. SCSI.
[20] D. R. Jefferson. Virtual time. ACM Trans. Program. Lang. Syst., 7(3):404-425, 1985.

[21] M. Jeschke, R. Ewald, A. Park, R. Fujimoto, and A. M. Uhrmacher. A parallel and distributed discrete event approach for spatial cell-biological simulations. SIGMETRICS Perform. Eval. Rev., 35(4):22-31, 2008.

[22] G. Karypis and V. Kumar. A Fast and High Quality Multilevel Scheme for Partitioning Irregular Graphs. SIAM J. Sci. Comput., 20(1):359-392, 1998.

[23] H. Leong, D. Agrawal, and J. Agre. Using message semantics to reduce rollback in the time warp mechanism. volume 725 of Lecture Notes in Computer Science, pages 309-323. Springer Berlin Heidelberg, 1993.

[24] J. Liu and D. M. Nicol. Lookahead revisited in wireless network simulations. In Proceedings of 16th Workshop on Parallel and Distributed Simulation, pages 79-88, Arlington, VA, USA, May 12-15 2002. IEEE.

[25] B. D. Lubachevsky. Efficient parallel simulations of dynamic ising spin systems. J. Comput. Phys., 75(1):103-122, 1988.

[26] J. Misra. Distributed discrete-event simulation. ACM Comput. Surv., 18(1):39-65, 1986.

[27] A. Pellegrini, R. Vitali, S. Peluso, and F. Quaglia. Transparent and efficient shared-state management for optimistic simulations on multi-core machines. In Proceedings of the 20th International Symposium on Modeling, Analysis Simulation of Computer and Telecommunication Systems, pages 134-141, Arlington, VA, USA, August 7-9 2012. IEEE.

[28] M. Rathinam, P. W. Sheppard, and M. Khammash. Efficient computation of parameter sensitivities of discrete stochastic chemical reaction networks. $J$. Chem. Phys., 132(3):034103, Jan. 2010.

[29] P. L. Reiher, F. Wieland, and D. Jefferson. Limitation of optimism in the time warp operating system. In Proceedings of the 21st Winter Simulation Conference, pages 765-770, Washington, D.C., USA, Dec. 4-6 1989. ACM.

[30] R. B. Schinazi. Predator-prey and host-parasite spatial stochastic models. Ann. Appl. Probab., 7(1):1-9, 1997.

[31] L. M. Sokol, D. P. Briscoe, and A. P. Wieland. MTW: A strategy for scheduling discrete simulation events for concurrent execution. In Proceedings of the SCS Multiconference on Distributed Simulation, pages 34-42, San Diego, CA, USA, Feb. 3-5 1988. SCSI.

[32] S. Srinivasan and P. F. R. Jr. Elastic time. ACM Trans. Model. Comput. Simul., 8(2):103-139, 1998.

[33] J. S. Steinman. Breathing time warp. In Proceedings of the 7th Workshop on Parallel and Distributed Simulation, pages 109-118, San Diego, CA, USA, May 16-19 1993. ACM.

[34] B. Wang, B. Hou, F. Xing, and Y. Yao. Abstract next subvolume method: A logical process-based approach for spatial stochastic simulation of chemical reactions. Comput. Biol. Chem., 35(3):193-198, 2011.

[35] J. Wang, D. Jagtap, N. B. Abu-Ghazaleh, and D. Ponomarev. Parallel discrete event simulation for multi-core systems: Analysis and optimization. IEEE Trans. Parallel Distrib. Syst., 25(6):1574-1584, 2014. 